Voix et Images

volxetimages

\title{
Narrations contemporaines au Québec et en France : regards croisés
}

\section{Robert Dion et Andrée Mercier}

Volume 36, numéro 1 (106), automne 2010

Narrations contemporaines au Québec et en France : regards croisés

URI : https://id.erudit.org/iderudit/045231ar

DOI : https://doi.org/10.7202/045231ar

Aller au sommaire du numéro

Éditeur(s)

Université du Québec à Montréal

ISSN

0318-9201 (imprimé)

1705-933X (numérique)

Découvrir la revue

Citer ce document

Dion, R. \& Mercier, A. (2010). Narrations contemporaines au Québec et en

France : regards croisés. Voix et Images, 36(1), 7-11.

https://doi.org/10.7202/045231ar d'utilisation que vous pouvez consulter en ligne.

https://apropos.erudit.org/fr/usagers/politique-dutilisation/ 


\title{
NARRATIONS CONTEMPORAINES AU QUÉBEC ET EN FRANCE : REGARDS CROISÉS
}

\author{
$+++$ \\ ROBERT DION \\ Université du Québec à Montréal \\ ANDRÉE MERCIER \\ Université Laval
}

C'est à la rencontre du contemporain, du narratif et du rapport Québec-France que convie ce dossier de la revue Voix et Images. Il s'agit d'abord d'envisager la littérature contemporaine et de tenter d'en rendre compte. Par «contemporain », on entend assez spontanément une période dont la borne initiale est le plus souvent fixée au début de la décennie $1980^{1}$, ce qui instaure un rapport de proximité qui rend instable ou à tout le moins questionnable une telle périodisation. L'examen du discours critique montre cependant que l'idée de contemporain en arrive également à agir de plus en plus comme un principe structurant, comme un mode d'appréhension et de compréhension des pratiques littéraires. Le contemporain apparaît alors comme un objet construit (ou, mieux, en construction), plus exactement comme une configuration esthétique où formes, questions et enjeux s'articulent. Si la revue Voix et Images contribue elle-même à ce travail d'exploration et d'élaboration du territoire contemporain en consacrant plusieurs de ses dossiers à des auteurs actifs - sans compter ses chroniques particulièrement attentives aux nouvelles parutions -, ce dossier invite, quant à lui, à une réflexion explicite et concertée sur le contemporain ${ }^{2}$.

Le narratif occupera ici une position privilégiée. En effet, il semble difficile, en quelques articles, de traverser autrement que de façon superficielle l'ensemble de la production littéraire; d'où l'intérêt de se donner un angle précis d'observation. Plus essentiellement, c'est aussi prendre acte d'une dominante: le narratif, notamment grâce au genre du roman mais aussi de la nouvelle, occupe une très large part du

$$
+++
$$

1 Plusieurs s'entendent sur cette borne initiale qui viendrait marquer le changement de la production littéraire québécoise. Signalons entre autres : Pierre Nepveu, L'écologie du réel. Mort et naissance de la littérature québécoise contemporaine, Montréal, Boréal Express, coll. «Papiers collés», 1988, 243 p.; Jacques Allard, Le roman mauve. Microlecture de la fiction récente au Québec, Montréal, Québec Amérique, 1997, 392 p.; Hans-Jürgen Greif et François Ouellet, La littérature québécoise, 1960-2000, Québec, L'instant même, coll. «Connaître», 2004,116 p. ; Michel Biron, François Dumont et Élisabeth Nardout-Lafarge, avec la collaboration de Martine-Emmanuelle Lapointe, Histoire de la littérature québécoise, Montréal, Boréal, 2007, 689 p. 2 Sur la notion même de contemporain, et pour connaître l'état de la réflexion sur la production contemporaine au Québec, on lira avec profit René Audet, "Le contemporain. Autopsie d'un mort-né», René Audet (dir.), Enjeux du contemporain. Études sur la littêrature actuelle, Québec, Nota bene, coll. «Contemporanéités», 2009, p. 7-19 et Viviane Asselin, «Fuites. Prosopopée de la recherche sur le roman québécois contemporain», paru dans le même ouvrage, p. 21-47. 
marché éditorial, à laquelle s'ajoute l'espace important des écritures autobiographiques et biographiques ${ }^{3}$. Il constitue par ailleurs une zone d'interaction particulièrement riche entre les pratiques littéraires et plus largement artistiques. C'est donc moins l'hégémonie d'un genre qui est en cause - et qui nous intéresse - que la transversalité et la portée du narratif dans l'écriture contemporaine, aussi bien littéraire que critique ou théorique. Le narratif non seulement domine, mais il détermine, surtout, et occupe la littérature et les réflexions sur le contemporain.

Enfin, c'est le rapport Québec-France qui oriente de façon particulière l'ensemble des études réunies dans ce dossier. Quel est le rapport de la littérature narrative québécoise contemporaine à la littérature française actuelle? Par cette question, il ne s'agit pas de relancer la discussion sur la quête de légitimité de la littérature québécoise à l'égard de la littérature française. Si l'institution littéraire et le marché du livre français exercent toujours un certain attrait et disposent d'une valeur symbolique et marchande forte, il reste que le rapport Québec-France a bien changé. Comme le soulignent les auteurs de l'Histoire de la littérature québécoise,

[...] l'écrivain québécois né vers 1960 et formé dans les tout nouveaux cégeps n'a plus du tout le même rapport à la France que l'écrivain né en 1940 et formé dans les collèges classiques. [...] L'écrivain québécois contemporain ne se situe plus seulement, ni même d'abord, par rapport à la littérature française, mais par rapport aux littératures dites étrangères, à commencer par celles d'Amérique, qui lui sont géographiquement proches ${ }^{4}$.

Dans son analyse du roman québécois contemporain, André Lamontagne va dans le même sens. Observant la diversification de la référence littéraire, il constate que, lorsqu'elle est convoquée, la littérature française se voit souvent désacralisée et démythifiée, comme c'est le cas dans Maryse de Francine Noël ${ }^{5}$. En fait, on pourrait se demander, à considérer les travaux des dernières décennies qui ont été attentifs surtout à interroger l'américanité, passée et présente, de notre littérature, de même que «les espaces hétérogènes de l'altérité ${ }^{6}$ ", dont celui des écritures migrantes, s'il y a toujours des points de contact avec la "francité». Les chemins que suivent les deux littératures se sont-ils à ce point séparés que les repères esthétiques et poétiques de l'une soient devenus à peu près invisibles pour l'autre? Y a-t-il eu un décrochage irréversible?

$$
++
$$

3 On pourrait bien sûr montrer que le narratif excède les pratiques narratives et essaime vers la poésie et le théâtre; mais ce serait un autre sujet, sous-tendu par d'autres enjeux : dynamique des genres, sociopoétique, par exemple. Sur la transversalité du narratif, voir l'ouvrage La narrativité contemporaine au Québec, vol. 1, La littérature et ses enjeux narratifs, René Audet et Andrée Mercier (dir.) et vol. 2, Le théâtre et ses nouvelles dynamiques narratives, Chantal Hébert et Irène Perelli-Contos (dir.), Québec, Presses de l'Université Laval, 2004. 4 Michel Biron, François Dumont et Élisabeth Nardout-Lafarge, Histoire de la littérature québécoise, p. 533. 5 André Lamontagne, Le roman québécois contemporain. Les voix sous les mots, Saint-Laurent/Québec, Fides, coll. «Nouvelles études québécoises», 2004, 281 p. Voir entre autres p. 98.6 Expression empruntée à Józef Kwaterko dans son avant-propos à l'ouvrage Imaginaire du roman québécois contemporain. Actes du colloque (Petr Kyloušek, Max Roy et Józef Kwaterko [dir.], Brno/Montréal, Masarykova univerzita/Université du Québec à Montréal, Figura, Centre de recherche sur le texte et l'imaginaire, 2006, p. 9). 
L'angle comparatiste que nous proposons vise donc à examiner la relation entre les deux littératures à l'aune du narratif et du contemporain. Cela veut d'abord dire que c'est le rapport entre la production actuelle aussi bien québécoise que française qui sera privilégié. Y a-t-il des points de convergence entre la production narrative française contemporaine et celle du Québec? Ces convergences concernent-elles des ensembles d'œuvres ou sont-elles au contraire très localisées? Si l'on considère le discours théorique français à travers lequel on a souvent lu et interprété la littérature québécoise au temps du nouveau roman ou de Tel Quel, ses propositions actuelles sur le contemporain, la modernité ou la postmodernité se montrent-elles pertinentes pour lire les œuvres narratives québécoises d'aujourd'hui ? La fécondité des échanges entre chercheurs québécois et français sur la production actuelle des deux côtés de l'Atlantique nous incite en tout cas à croire que les ponts n'ont pas été tout à fait coupés.

Poser la question de la relation entre les deux littératures à notre époque, c'est également se situer à un moment que Pierre Nepveu, dans L'écologie du réel, qualifiait de «mort de la littérature québécoise», moment associé à la fin du projet national «fondé sur une mémoire collective et une visée totalisante ${ }^{7}$ » qui avait animé la Révolution tranquille, cet «âge de la poésie ${ }^{8}$ ». Caractérisée plutôt par l'éclatement et par la pluralité des points de référence, la littérature québécoise contemporaine se trouve alors, selon Nepveu, à travailler à une redéfinition du lieu menant à un nouvel espace esthétique ${ }^{9}$. C'est dire que le rapport Québec-France, en contexte contemporain, tend à se définir en dehors de l'idée de centre et d'origine, de même que de littérature nationale, qui l'ont pendant longtemps déterminé. De fait, la littérature contemporaine appelle volontiers, semble-t-il, une perspective ouverte, une lecture à échelle plus grande ${ }^{10}$, celle que désigne la notion de «littérature mondiale», dans le cadre de laquelle l'exercice de comparaison ne tend pas essentiellement à rattacher les différences ou les convergences observées aux singularités nationales, mais cherche à «réaménager la cartographie des centres et des périphéries ${ }^{11}$ » et à penser les littératures dans la bibliothèque universelle. Comme le soulignent Christophe Pradeau et Tiphaine Samoyault à propos de leur ouvrage Où est la littérature mondiale?, une telle perspective exige cependant de poser le lieu à partir duquel on lit les littératures et on les compare ${ }^{12}$. Dans

$$
+++
$$

7 Pierre Nepveu, L'écologie du réel, p. 14. 8 Nepveu précise que cet «âge de la poésie» de la Révolution tranquille serait plus justement une mutation ou une crise de la poésie (ouvr. cité, p. 22). Lise Gauvin et Franca Malcato-Fazoni intituleront leur ouvrage consacré à la littérature des années 1980 L'âge de la prose, signalant bien par là la part et le rôle dominants tenus désormais par le narratif : «Par rapport aux décennies précédentes, celle des années 1980 frappe par la quantité et la qualité de ses romanciers, "nouvelliers", "récitants" de toutes tendances. Si on peut attribuer à l'ensemble des années 1960 le titre du recueil de Giguère, L'âge de la parole, les années 1980 sont, incontestablement, "l'âge de la prose". » (Lise Gauvin, «Introduction», Lise Gauvin et Franca Malcato-Fazoni [dir.], L'âge de la prose. Romans et récits québécois des années 1980, Rome/Montréal, Bulzoni/VLB éditeur, 1992, p. 10. 9 On peut relire à ce sujet la conclusion de L'écologie du réel. 10 Nous reprenons ici une expression de René Audet dans l'article qu'il signe dans ce dossier. $\mathbf{1 1}$ Christophe Pradeau et Tiphaine Samoyault, Où est la littérature mondiale?, http://www.litterature-et-histoires.univ-paris8.fr/ index.php?option=com_content\&view=article\&id=80:christophe-pradeau-et-tiphaine-samoyault-dir-ou-est-lalitterature-mondiale-\&catid=34:actualites-des-publications\&Itemid=59 (site consulté le 25 septembre 2010). 12 Site précédemment cité. Nous renvoyons également à l'ouvrage dirigé par Christophe Pradeau et Tiphaine 
le cadre de ce dossier, c'est résolument à partir des problématiques et des œuvres de la littérature québécoise que les relations seront établies.

Si les différents articles rassemblés ici prennent acte des déterminants culturels spécifiques aux littératures québécoise et française, ce sont moins les paramètres sociohistoriques et institutionnels qui seront considérés et approfondis que les poétiques et les esthétiques de ces deux ensembles. L'attention sera donc accordée au travail des formes, de l'écriture et de la composition, aux poétiques du récit et du littéraire, ainsi qu'aux enjeux esthétiques qui y sont rattachés. Il s'agit de rendre compte du contemporain par la comparaison ou le "compagnonnage ${ }^{13}$ " des œuvres narratives, mais aussi des discours critiques sur les pratiques littéraires. On constatera au fil des études un certain nombre de notions ou d'éléments récurrents qui caractérisent ou préoccupent la littérature narrative contemporaine en dépit des différences qui éloignent les pratiques québécoise et française: la fragmentation et l'éclatement textuel ou énonciatif, le minimalisme, la question du genre, le registre de l'intime et celui de l'autobiographie, la filiation, le discours du savoir et des valeurs (en mode sérieux ou ludique), la métatextualité, les modes de la représentation, etc. Ces éléments se verront saisis sous des angles spécifiques aptes à leur donner sens et à permettre une lecture croisée des deux productions.

Ainsi, dans l'article de René Audet, l'éclatement et la fragmentation qui, au Québec et en France, donnent lieu à des manifestations textuelles extrêmement variées, sont considérés moins comme des états multiples et hétérogènes que comme un processus général et déterminant de la prose narrative contemporaine. Envisagée comme une apparente "impassibilité ${ }^{14}$ » et une réduction narrative du côté français, définie plutôt par son caractère intimiste du côté québécois, la notion de minimalisme à laquelle s'intéressent Marie-Pascale Huglo et Kimberley Leppik conduit à désigner une modalité de la narrativité contemporaine fondée sur une tension entre une succession d'incidents et leur portée significative. Dans les «récits du lieu» de Pierre Morency (L'œil américain) et de Pierre Bergounioux (Le grand sylvain), Élisabeth Nardout-Lafarge dégage des pratiques distinctes de la narrativité, des formes du récit et du discours des savoirs, pratiques et formes engagées cependant dans une entreprise commune de narrativisation d'une présence au monde et d'un rapport au temps. Francis Langevin aborde la représentation de la région (au Québec) et de la province (en France) par la question du devenir du régionalisme littéraire, vu comme une rhétorique et une négociation de régimes de valeurs, produisant dans chacun des corpus comparés (romans de Sébastien Chabot, d'Éric Dupont et de Christine Eddie pour le Québec; récits de Pierre Michon, de Pierre Bergounioux et de Richard Millet pour la France) des modulations plutôt contrastées du rapport au littéraire, au monde et à la textualité. Barbara Havercroft s'attache pour sa part aux stratégies discursives de l'écriture de la perte, et plus exactement

Samoyault, Où est la littérature mondiale?, Saint-Denis, Presses universitaires de Vincennes, coll. «Essais et savoirs", 2005, 150 p. 13 Pour reprendre l'expression proposée par Élisabeth Nardout-Lafarge dans l'article qu'elle signe dans ce dossier. 14 Toute une école tournant autour des Éditions de Minuit (Oster, Toussaint, Gailly, etc.) est désignée par les journalistes comme celle des «romanciers impassibles». 
du deuil, auxquelles ont recours les œuvres de Denise Desautels (Ce désir toujours: un abécédaire) et de Laure Adler (À ce soir), de façon à montrer la richesse des stratégies langagières et rhétoriques d'une poétique générale du deuil animée par le désir de dire l'inexprimable. Enfin, dans l'article de Frances Fortier, un vaste ensemble de romans québécois et français aux ambitions et aux esthétiques très variées (Hier de Nicole Brossard, Maleficium de Martine Desjardins, L'imitation du bonheur de Jean Rouaud, Cinéma de Tanguy Viel, etc.) sont réunis par une commune problématisation de l'autorité narrative - paradigme qui, quoique renvoyé au Québec et en France à des conceptions différentes du fait littéraire et de sa précarisation, se voit plutôt envisagé ici comme une mise en relief et une exploration du procès d'adhésion au raconté.

Résolument inspiré par la poétique, ce regard croisé sur les narrations contemporaines au Québec et en France n'épuise évidemment pas les possibles points de comparaison et de relance entre les deux littératures. Il montre cependant la pertinence de cette mise en rapport - et de toute mise en rapport - pour saisir les enjeux des pratiques littéraires contemporaines, ici et ailleurs. 